\title{
Social Support from Developmental Contexts and Adolescent Substance Use and Well-Being: A Comparative Study of Spain and Portugal
}

\author{
Antonia Jiménez-Iglesias ${ }^{1}$, Inês Camacho ${ }^{2}$, Francisco Rivera' ${ }^{1}$, Carmen Moreno ${ }^{1}$ and \\ Margarida Gaspar de Matos $^{2}$ \\ 1 Universidad de Sevilla (Spain) \\ 2 Universidade de Lisboa (Portugal)
}

\begin{abstract}
The aim of this study was to analyse the contribution of social support from family, friend and school (teacher and classmate) contexts in substance use (tobacco and alcohol use) and well-being (life satisfaction and health-related quality of life). Participants were 5,784 Portuguese and 22,610 Spanish adolescents aged 11 to 16 years, from the 2014 edition of the Health Behavior in School-aged Children (HBSC) study in Portugal and Spain. Results showed that for a higher life satisfaction, family $\left(p<.001\right.$, partial $\left.\eta^{2}=.032\right)$, teacher $\left(p<.001\right.$, partial $\left.\eta^{2}=.018\right)$ and classmate $(p<.001$, partial $\left.\eta^{2}=.031\right)$ support were important in Portugal, and family $\left(p<.001\right.$, partial $\left.\eta^{2}=.056\right)$ and friend $(p<.001$, partial $\left.\eta^{2}=.015\right)$ support in Spain. Similarly, for a better health-related quality of life, all the social support variables were relevant in Portugal (family: $p<.001$, partial $\eta^{2}=.063$; teacher: $p<.001$, partial $\eta^{2}=.032$; classmate: $p<.001$, partial $\eta^{2}=.054$; friend: $p<.001$, partial $\eta^{2}=.034$ ) and in Spain (family: $p<.001$, partial $\eta^{2}=.054$; teacher: $p<.001$, partial $\eta^{2}=.014$; classmate: $p<.001$, partial $\eta^{2}=.018$; friend: $p<.001$, partial $\left.\eta^{2}=.040\right)$. In contrast, only family support $\left(p<.001\right.$, partial $\left.\eta^{2}=.014\right)$ was relevant in Portugal for tobacco use. Therefore, social support was more relevant for adolescent well-being than for adolescent substance use, and the most relevant source of support was family support, in both Spain and Portugal.
\end{abstract}

Received 29 July 2016; Revised 23 October 2017; Accepted 25 October 2017

Keywords: adolescence, developmental contexts, social support, substance use, well-being.

Family, school and community (peers, leisure, and neighborhood) are primary developmental contexts for adolescents. Research and intervention in these contexts are very important to prevent externalized and internalized problems as well as to promote health and well-being in these contexts (Stattin \& Kerr, 2009). For example, positive parent-child relationships result in lower substance use (Johnson, McBride, Hopkins, \& Pepper, 2014), such as alcohol and tobacco use (Piko \& Balázs, 2012). In a qualitative study by Navarro et al. (2017), relationships with

Correspondence concerning this article should be addressed to Antonia Jiménez-Iglesias. Departamento de Psicología Evolutiva y de la Educación. Calle Camilo José Cela, s/n. 41018. Sevilla (Spain). Phone: +34-955420544

E-mail: amjimigl@us.es

The HBSC study in Portugal was supported by the Direção-Geral da Saúde-Ministério da Saúde. The HBSC study in Spain was supported by the Ministerio de Sanidad, Servicios Sociales e Igualdad; Antonia Jiménez-Iglesias' work was supported by the V Plan Propio de Investigación de la Universidad de Sevilla 2014, under the action “II.5B Contrato de acceso al Sistema Español de Ciencia, Tecnología e Innovación para el Desarrollo del Programa Propio de $\mathrm{I}+\mathrm{D}+\mathrm{i}$ de la Universidad de Sevilla" and by the Ministerio de Educación, Cultura y Deporte "Programa Estatal de Promoción del Talento y su Empleabilidad en I+D+i, Subprograma Estatal de Movilidad, del Plan Estatal de Investigación Científica y Técnica y de Innovación 2013-2016" (Referencia: JC2015-00399) family and friends were considered to be the most important for well-being, confirmed by other studies indicating that family and peer relationships have a positive influence on adolescent well-being (Tomé, Matos, Camacho, Simões, \& Diniz, 2012; Williams \& Anthony, 2015). Additionally, a positive school climate promotes healthy development, with lower drug use and higher mental health (LaRusso, Romer, \& Selman, 2008).

\section{Social support from developmental contexts}

This study examines family, school and peer contexts. Specifically, it analyses the perceived social support from these developmental contexts and the contribution of the family, teachers, classmates and friends support in adolescent substance use and well-being.

Social support is a relevant aspect of each context, which in general refers to the help and emotional support that adolescents may have from different sources, such as family, friends, teachers or classmates.

How to cite this article:

Jiménez-Iglesias, A., Camacho, I., Rivera, F., Moreno, C., \& Matos, M. G. (2017). Social support from developmental contexts and adolescent substance use and well-being: A comparative study of Spain and Portugal. The Spanish Journal of Psychology, 20. e64. Doi:10.1017/sjp.2017.62 
Perceptions of social support from different contexts vary according to age and gender. Girls perceive higher friend support, teacher support (Bokhorst, Sumter, \& Westenberg, 2010) and classmate support than boys (Bokhorst et al., 2010; Hombrados-Mendieta, GomezJacinto, Dominguez-Fuentes, Garcia-Leiva, \& CastroTravé, 2012). In addition, younger adolescents perceive similarly important parental and friend support and classmate and teacher support (Bokhorst et al., 2010), and according to other research maternal support is the main source of support followed by paternal, classmate and teacher support (Hombrados-Mendieta et al., 2012). In contrast, older adolescents perceive friend support as more important than parental support (Bokhorst et al., 2010) and classmate support as similarly important to parental support (Bokhorst et al., 2010; Hombrados-Mendieta et al., 2012), whereas they perceive lower teacher support. This decrease of teacher support is probably due to the transition from primary to secondary school (Bokhorst et al., 2010). Regarding the differences between parental and friend support, during adolescence parental support decreases and friend support increases, finally reaching similar levels (Helsen, Vollebergh, \& Meeus, 2000). This could be related to the decrease of parental influence and the increase of friend influence during adolescence, although both are similarly relevant towards late adolescence (De Goede, Branje, Delsing, \& Meeus, 2009).

In addition, substance use increases during adolescence (Sánchez-Queija, Oliva, Parra, \& Camacho, 2015), it is higher in older than in younger adolescents (Jiménez-Iglesias, Moreno, Rivera, \& García-Moya, 2013), and boys' substance use increases more than girls' substance use along adolescence (Sánchez-Queija et al., 2015). Regarding well-being, girls and older adolescents show lower levels of well-being (Erhart et al., 2009; Ramos, Moreno, Rivera, Matos, \& Morgan, 2012). Perceived social support is associated with child and adolescent well-being and it seems that the association between social support and well-being increases with age, being greater in girls than in boys (Chu, Saucier, \& Hafner, 2010). In contrast, poor social support is a risk factor for adolescent mental health problems (Ravens-Sieberer, Erhart, Gosch, Wille, \& The European KIDSCREEN Group, 2008).

\section{Family support, adolescent substance use and well-being}

Parental support is the source of support most strongly associated with mental health, particularly with positive life satisfaction (Stewart \& Suldo, 2011), it is considered the best indicator of lower emotional problems throughout adolescence and it seems to influence the effect of friend support. Specifically, in adolescents with higher parental support, friend support had a slightly positive effect, whereas in adolescents with lower parental support, friend support had a negative effect (Helsen et al., 2000).

Family support positively influences adolescents' subjective well-being (Leme, Del Prette, \& Coimbra, 2015; Williams \& Anthony, 2015), such as life satisfaction (Gutiérrez \& Gonçalves, 2013; Rodríguez-Fernández, Droguett, \& Revuelta, 2012). Similarly, perceived support from adult family members is associated with fewer depressive symptoms (Tummala-Narra \& SathasivamRueckert, 2013) and parental support is associated with fewer emotional symptoms (Demaray \& Malecki, 2002). Moreover, parental support is negatively related to substance use (Wills, Resko, Ainette, \& Mendoza, 2004) and maternal support is associated with lower substance use (Branstetter, Low, \& Furman, 2011). In fact, substance use is lower in boys and girls who had caring and supportive relationships in their families during childhood, and cohesive family context throughout adolescence and emerging adulthood (Sánchez-Queija et al., 2015).

\section{Friend support, adolescent substance use and well-being}

Friend support positively influences adolescents' subjective well-being (Leme et al., 2015; Williams \& Anthony, 2015), such as life satisfaction (RodríguezFernández et al., 2012). However, others studies have found the contrary, showing that friend support did not influence on life satisfaction (Gutiérrez \& Gonçalves, 2013). In the case of substance use, it has been found that friend support is not associated with substance use (Branstetter et al., 2011), and in contrast that peer support is positively related to substance use since peer group may favor risk behavior and have more positive substance use attitudes (Wills et al., 2004).

\section{School support, adolescent substance use and well-being}

Concerning school, classmate support has been associated with fewer emotional symptoms (Demaray \& Malecki, 2002), lower internalizing behavior and positive life satisfaction, and although teacher support did not associate with these variables, it did however with lower externalizing behavior (Stewart \& Suldo, 2011) and it indirectly influenced life satisfaction mediated by satisfaction with school (Gutiérrez \& Gonçalves, 2013). In addition, other studies have found that perceived support from adults in the school context (among them teachers) is not associated with depressive symptoms, probably because adolescents perceive adults at school as sources of support for academic issues and not for emotional issues (Tummala-Narra \& SathasivamRueckert, 2013). Possibly for similar reasons, in a study by Leme et al. (2015), teacher support did not influence adolescents' subjective well-being. However, other studies have found that teacher support is positively 
associated with subjective well-being (García-Moya, Brooks, Morgan, \& Moreno, 2015; Suldo et al., 2009) and low teacher support, among others school-related aspects, implies low subjective and social well-being (Pilkauskaite-Valickiene \& Gabrialaviciute, 2015).

In fact, a meta-analysis found that support from teachers and school personnel had the highest association with well-being, followed by family support, while friend support and other support had the lowest association (Chu et al., 2010). In addition, it has been found that adolescents with higher teacher support perceive their schools as having a more respectful and healthy climate and this promotes lower substance use as well as higher social belonging and fewer depressive symptoms (LaRusso et al., 2008).

\section{Social support in Spain and Portugal}

Social support seems to be an important factor for adolescent development and it is therefore of great interest to know how perceived support in different contexts, such as family, school and peers, contributes to their development in two similar countries, like Spain and Portugal.

In general, Portugal and Spain share many cultural and political similarities, which make it relevant to carry out this study in both countries. For example, Spain and Portugal are two of the European countries that assign less money to family-related policies (Instituto de Política Familiar, 2014); the education system is similar in both countries, Portuguese and Spanish adolescents' perception about school generates concern (Inchley et al., 2016) and the model for the provision of school health services is community-based (children and adolescents are supervised by health professionals working in health centers separated from the schools) in both Spain and Portugal (Pommier et al., 2010). Additionally, adolescents from Spain and Portugal show a positive perception of their families, parental support and good relationships, are generally involved in unproblematic peer group, as well as being satisfied with aspects of their school context (Serrano, El-Astal, \& Faro, 2004).

\section{Aims and hypotheses}

The aim of this study was to analyze the role of social support from the main developmental contexts (family, school-teacher and classmate- and friend) in adolescent substance use (tobacco and alcohol use) and well-being (life satisfaction and health-related quality of life) in Portugal and Spain. Analyses were performed after deleting the effects of gender and age since, as has been indicated above, these demographic variables are related to social support, substance use and well-being and could affect the relationships between them. It was expected that all the sources of social support would be relevant for adolescent substance use and well-being, especially family support, and the social support from the main developmental contexts would be particularly important for adolescent well-being. Additionally, similar results were expected between Spain and Portugal due to similarities in both countries.

\section{Method}

\section{Participants}

The participants were boys and girls aged 11 to 16 years from Portugal and Spain who took part in the 2014 edition of the Health Behavior in School-aged Children (HBSC) study, a WHO collaborative cross-national study, in both countries. Specifically, the sample consisted of 5,784 Portuguese adolescents and 22,610 Spanish adolescents (see table 1).

In Portugal, participants were randomly chosen from 36 national vertical clusters of schools, in a total of 473 classes, in a national sample geographically stratified by Education Regional Divisions (Matos, Simões, Camacho, Reis, \& Equipa Aventura Social, 2015). In Spain, participants were selected through a random multistage sampling procedure stratified by conglomerates taking into account age, habitat (rural or urban) and type of educational centre (public or private) (Moreno et al., 2016). Both samples were nationally representative regarding those ages.

\section{Measures}

The measures used in this study come from the 2014 edition of the HBSC questionnaire. In order to ensure that the same variables are measured in all countries, the HBSC study recommends carrying out a specific and critical translation process of the questionnaire: first, the measures are translated from English to national languages (Portuguese and Spanish in the case of this study); second, they are back-translated into English by a different translator; thirdly, the backtranslations are reviewed by the national teams; and finally, the back translated measures are reviewed by the Translation Group Reviewer of the HBSC study

Table 1. Study sample

\begin{tabular}{lllllll}
\hline & & \multicolumn{2}{l}{ Portugal } & & \multicolumn{2}{l}{ Spain } \\
\cline { 3 - 4 } \cline { 7 - 8 } \cline { 6 - 7 } & & $N$ & $\%$ & & $N$ & $\%$ \\
\hline \multirow{2}{*}{ Gender } & Boy & 2,756 & $47.65 \%$ & & 11,204 & $49.55 \%$ \\
& Girl & 3,028 & $52.35 \%$ & & 11,406 & $50.45 \%$ \\
& $11-12$ years & 1,776 & $30.71 \%$ & & 7,356 & $32.53 \%$ \\
& $13-14$ years & 2,331 & $40.30 \%$ & & 7,691 & $34.02 \%$ \\
& $15-16$ years & 1,677 & $28.99 \%$ & & 7,563 & $33.45 \%$ \\
\hline
\end{tabular}


(Aleman-Diaz et al., 2013). For this study, the selected variables were as follows:

Demographic variables:

- Country: Portugal and Spain.

- Gender: boys and girls.

- Age: 11-12, 13-14 and 15-16 years.

Adolescents' perceptions about social support variables:

- Family support: this variable was the family subscale of the Multidimensional Scale of Perceived Social Support (MSPSS): 'my family really tries to help me/ I get the emotional help and support I need from my family / I can talk about my problems with my family / my family is willing to help me make decisions'. Cronbach's coefficient alpha of this subscale was .87, indicating good internal consistency (Zimet, Dahlem, Zimet, \& Farley, 1988). In this study, the values of Cronbach's alpha were .95 in Portugal and .93 in Spain. The scale ranges from 1 (very strongly disagree) to 7 (very strongly agree). - Teacher support: this variable was developed by the HBSC study and measures the support from teachers through a scale with three items 'I feel that my teachers accept me as I am/ I feel that my teachers care about me as a person/ I feel a lot of trust in my teachers'. The scale has a high internal reliability with a Cronbach's alpha of .82 (Rasmussen et al., 2013), similar to the values obtained in this study of .84 in Portugal and Spain. The scale ranges from 1 (strongly agree) to 5 (strongly disagree).

- Classmate support: this variable was developed by the HBSC study and assesses the support from classmate through a scale with three items 'the students in my class(es) enjoy being together/ most of the students in my class(es) are kind and helpful/ other students accept me as I am'. The scale has a high internal reliability with a Cronbach's alpha of .74 (Rasmussen et al., 2013), and in this study the values were similar with .76 in Portugal and .80 in Spain. The scale ranges from 1 (strongly agree) to 5 (strongly disagree).

- Friend support: this variable was the friends subscale of the Multidimensional Scale of Perceived Social Support (MSPSS): 'my friends really try to help me/ I can count on my friends when things go wrong/ I have friends with whom I can share my joys and sorrows / I can talk about my problems with my friends'. Cronbach's coefficient alpha of this subscale was .85, showing good internal consistency (Zimet et al., 1988). In this study, the values of Cronbach's alpha were .96 in Portugal and .93 in Spain. The scale ranges from 1 (very strongly disagree) to 7 (very strongly agree).

Adolescent substance use variables:

- Tobacco use: this variable was adapted from Monitoring the Future-study and the European
School Survey Project on Alcohol and Other Drugs (ESPAD), it analyses the prevalence over the last 30 days of tobacco use: 'On how many days (if any) have you smoked cigarettes? In the last 30 days', and it is a good measure of smoking prevalence (Hublet \& Fotiou, 2013). The values range from 0 (never) to 30 (30 days or more).

- Alcohol use: this variable was adapted from Monitoring the Future-study and the European School Survey Project on Alcohol and Other Drugs (ESPAD), it examines the prevalence over the last 30 days of alcohol use: 'On how many days (if any) have you drunk alcohol? In the last 30 days', and it is a good measure of frequency of usual drinking (Kuntsche et al., 2013). The values range from 0 (never) to 30 (30 days or more).

Adolescent well-being variables:

- Life satisfaction: this variable was measured through the Cantril Ladder (Cantril, 1965), which it is good measure of general life satisfaction: 'Here is a picture of a ladder. The top of the ladder " 10 " is the best possible life for you and the bottom " 0 " is the worst possible life for you. In general, where on the ladder do you feel you stand at the moment?'. This item ranges from 0 (worst possible life) to 10 (best possible life).

- Health-related quality of life: this variable was taken from the KIDSCREEN-10 index questionnaire, which has a good reliability $(\alpha=.82)$ (RavensSieberer \& The KIDSCREEN group Europe 2006). The KIDSCREEN-10 index is composed of 10 items about physical, psychological and social aspects of subjective health and well-being: 'Thinking about the last week... have you felt fit and well? / have you felt full of energy?/ have you felt sad?/ have you felt lonely?/ have you had enough time for yourself? / have you been able to do the things that you want to do in your free time? / have your parent(s) treated you fairly?/ have you had fun with your friends? / have you got on well at school?/ have you been able to pay attention?'. The scale ranges from 10 (never) to 50 (always). The values of Cronbach's alpha in this study were .82 in Portugal and Spain.

\section{Procedure}

\section{Data collection}

The study was approved by the Ethical Research Committee of the University of Seville and the Ethical Commission of the Hospital S. João do Porto and was authorized by the Portuguese Ministry of Education and the Spanish Ministry of Health, Social Services and Equality (Matos et al., 2015; Moreno et al., 2016).

The data collection in both Portugal and Spain was done through an online questionnaire that fulfilled the requirements of the HBSC study (Matos et al., 2015; 
Moreno et al., 2016): the adolescents must answer the questionnaire themselves; the anonymity and confidentiality of their answers must be guaranteed; and the questionnaires must be administered within the school context (Inchley et al., 2016).

\section{Statistical analysis}

The statistical analyses were performed using the IBM SPSS Statistics 23 program and were carried out separately for Portugal and Spain to analyze differences between both countries.

General Linear Models were used in order to control the effect of gender and age on the substance use and well-being variables and to save the unstandardized residual dependent variable. Following this, General Linear Models were performed to examine the role of social support in unstandardized residual variables of substance use and well-being after the effect of gender and age had been eliminated. $R^{2}$ (explained variance) values were used to inform about the relevance of the models, specifically using Cohen's criteria (1988), the relevance was considered negligible from 0 to .019 , small from .02 to .129 , medium from .13 to .259 , and large from .26 or greater. Partial eta squared (partial $\eta^{2}$ ) values were utilized to analyze the effect size of each variable, taking into account that values were considered negligible from 0 to .009 , small from .01 to .059 , medium from .06 to .149 , and large from .15 or greater (Cohen, 1988).

\section{Results}

General Linear Models of gender and age on the substance use variables were significant and with small relevance in Portugal and Spain for tobacco use (Portugal: $F(3,5623)=61.620, p<.001, R^{2}=.032$; Spain: $\left.F(3,19199)=212.521, p<.001, R^{2}=.032\right)$ and for alcohol use (Portugal: $F(3,5634)=64.083, p<.001$, $R^{2}=.033$; Spain: $\left.F(3,19121)=242.938, p<.001, R^{2}=.037\right)$. Similarly, General Linear Models of gender and age on the well-being variables were also significant with small relevance in Portugal and Spain for life satisfaction (Portugal: $F(3,5524)=87.999, p<.001, R^{2}=.046$; Spain $\left(F(3,18488)=484.230, p<.001, R^{2}=.073\right)$ and for healthrelated quality of life (Portugal: $F(3,5523)=230.582$, $p<.001, R^{2}=.111$; Spain: $F(3,16630)=770.190, p<.001$, $\left.R^{2}=.122\right)$.

On the other hand, General Linear Models used to analyze the role of social support in unstandardized residual variables of substance use and well-being after the effect of gender and age had been eliminated showed similar results in Spain and Portugal.

The model of social support from developmental contexts on tobacco use was significant and with small relevance in Portugal $(F(4,4913)=29.379, p<.001$, $\left.R^{2}=.023\right)$. All social support variables, except classmate support, were significant, but the effect size was only noticeable for family support $\left(p<.001\right.$, partial $\left.\eta^{2}=.014\right)$ with higher values favoring less tobacco use. On the contrary, in Spain the model was significant but the effect size did not reach a noticeable value $(F(4,15547)=59.086$, $\left.p<.001, R^{2}=.015\right)$. These results are shown in table 2 .

Regarding alcohol use (see table 3), the models were significant, but the effect sizes were negligible in Portugal $\left(F(4,4925)=12.724, p<.001, R^{2}=.010\right)$ and in Spain $\left(F(4,15502)=44.652, p<.001, R^{2}=.011\right)$.

Concerning the model of social support from developmental contexts on life satisfaction (higher values of social support involved higher life satisfaction), as is shown in table 4 , in Portugal it was significant with medium relevance $(F(4,4925)=200.255$, $\left.p<.001, R^{2}=.140\right)$. All the social support variables were significant and the effect sizes were small for family support $\left(p<.001\right.$, partial $\left.\eta^{2}=.032\right)$, teacher support $\left(p<.001\right.$, partial $\left.\eta^{2}=.018\right)$ and classmate support $\left(p<.001\right.$, partial $\left.\eta^{2}=.031\right)$, and the effect size was negligible for friend support $(p<.001$, partial $\left.\eta^{2}=.004\right)$. In Spain, this model was significant with medium relevance $(F(4,16126)=717.031, p<.001$, $\left.R^{2}=.151\right)$. Again, all the social support variables were significant, but the effect sizes were small only

Table 2. General Lineal Model of the social support from developmental contexts on tobacco use

\begin{tabular}{|c|c|c|c|c|c|c|}
\hline \multirow{4}{*}{ Corrected model } & \multicolumn{3}{|c|}{ Portugal } & \multicolumn{3}{|l|}{ Spain } \\
\hline & $F$ & $p$ & $R^{2}$ & $F$ & $p$ & $R^{2}$ \\
\hline & 29.379 & $<.001$ & .023 & 59.086 & $<.001$ & .015 \\
\hline & $F$ & $p$ & partial $\eta^{2}$ & $F$ & $p$ & partial $\eta^{2}$ \\
\hline Family support & 69.516 & $<.001$ & .014 & 50.321 & $<.001$ & .003 \\
\hline Teacher support & 24.049 & $<.001$ & .005 & 131.268 & $<.001$ & .008 \\
\hline Classmate support & 0.359 & .549 & .000 & 10.426 & .001 & .001 \\
\hline Friend support & 10.172 & .001 & .002 & 17.927 & $<.001$ & .001 \\
\hline
\end{tabular}


Table 3. General Lineal Model of the social support from developmental contexts on alcohol use

\begin{tabular}{|c|c|c|c|c|c|c|}
\hline \multirow{4}{*}{ Corrected model } & \multicolumn{3}{|c|}{ Portugal } & \multicolumn{3}{|l|}{ Spain } \\
\hline & $F$ & $p$ & $R^{2}$ & $F$ & $p$ & $R^{2}$ \\
\hline & 12.724 & $<.001$ & .010 & 44.652 & $<.001$ & .011 \\
\hline & $F$ & $p$ & partial $\eta^{2}$ & $F$ & $p$ & partial $\eta^{2}$ \\
\hline Family support & 32.308 & $<.001$ & .007 & 36.648 & $<.001$ & .002 \\
\hline Teacher support & 8.955 & .003 & .002 & 85.607 & $<.001$ & .005 \\
\hline Classmate support & 0.004 & .950 & .000 & 0.295 & .587 & .000 \\
\hline Friend support & 2.250 & .134 & .000 & 28.180 & $<.001$ & .002 \\
\hline
\end{tabular}

Table 4. General Lineal Model of the social support from developmental contexts on life satisfaction

\begin{tabular}{|c|c|c|c|c|c|c|}
\hline \multirow{4}{*}{ Corrected model } & \multicolumn{3}{|l|}{ Portugal } & \multicolumn{3}{|l|}{ Spain } \\
\hline & $F$ & $p$ & $R^{2}$ & $F$ & $p$ & $R^{2}$ \\
\hline & 200.255 & $<.001$ & .140 & 717.031 & $<.001$ & .151 \\
\hline & $F$ & $p$ & partial $\eta^{2}$ & $F$ & $p$ & partial $\eta^{2}$ \\
\hline Family support & 164.046 & $<.001$ & .032 & 960.107 & $<.001$ & .056 \\
\hline Teacher support & 88.895 & $<.001$ & .018 & 85.134 & $<.001$ & .005 \\
\hline Classmate support & 160.046 & $<.001$ & .031 & 128.205 & $<.001$ & .008 \\
\hline Friend support & 18.954 & $<.001$ & .004 & 253.057 & $<.001$ & .015 \\
\hline
\end{tabular}

for family support $\left(p<.001\right.$, partial $\left.\eta^{2}=.056\right)$ and friend support $\left(p<.001\right.$, partial $\left.\eta^{2}=.015\right)$, whereas for teacher support $\left(p<.001\right.$, partial $\left.\eta^{2}=.005\right)$ and classmate support $\left(p<.001\right.$, partial $\left.\eta^{2}=.008\right)$ they were negligible.

With respect to health-related quality of life, higher values of social support implied higher health-related quality of life. In Portugal, the model was significant with large effect size $(F(4,4925)=465.298, p<.001$, $\left.R^{2}=.274\right)$. The social support variables were also significant and the effect sizes were medium for family support $\left(p<.001\right.$, partial $\left.\eta^{2}=.063\right)$ and small for teacher support $\left(p<.001\right.$, partial $\left.\eta^{2}=.032\right)$, classmate support $\left(p<.001\right.$, partial $\left.\eta^{2}=.054\right)$ and friend support $(p<.001$, partial $\left.\eta^{2}=.034\right)$. Similarly, in Spain the model was significant with medium relevance $(F(4,14644)=1078.219$, $\left.p<.001, R^{2}=.228\right)$ and all the social support variables were significant with small effect size: family support $\left(p<.001\right.$, partial $\left.\eta^{2}=.054\right)$, teacher support $(p<.001$, partial $\left.\eta^{2}=.014\right)$, classmate support $(p<.001$, partial $\left.\eta^{2}=.018\right)$ and friend support $\left(p<.001\right.$, partial $\left.\eta^{2}=.040\right)$. The results are shown in table 5 .

\section{Discussion}

The purpose of this study was to know what sources of social support from the main developmental contexts (family, school-teacher and classmate- and friend) were relevant for substance use (tobacco and alcohol use) and well-being (life satisfaction and health-related quality of life) in Portuguese and Spanish adolescents. Analyses were performed after deleting the effect of gender and age. The results of this study partially agreed with its hypotheses.

Social support variables were not relevant for substance use, with the exception of family support for tobacco use in Portugal, showing that higher values of parental support imply less substance use, as confirmed by previous studies (Wills et al., 2004). These results could be due to the fact that although different developmental contexts are analyzed, the social support variable is only one aspect of each context and is probably not as relevant to substance use as it is for well-being. As was found by Branstetter et al. (2011), it would have been important to analyze different aspects of each context and not only one aspect, such as social support in the case of our study. Regarding friends, it is very likely that friends' substance use and the peer pressure to use substances have a clearer role in predicting adolescent substance use than friend support. Therefore, the results of this study do not prove that the developmental contexts analyzed are 
Table 5. General Lineal Model of the social support from developmental contexts on health-related quality of life

\begin{tabular}{|c|c|c|c|c|c|c|}
\hline \multirow{4}{*}{ Corrected model } & \multicolumn{3}{|l|}{ Portugal } & \multicolumn{3}{|l|}{ Spain } \\
\hline & $F$ & $p$ & $R^{2}$ & $F$ & $p$ & $R^{2}$ \\
\hline & 465.298 & $<.001$ & .274 & 1078.219 & $<.001$ & .228 \\
\hline & $F$ & $p$ & partial $\eta^{2}$ & $F$ & $p$ & partial $\eta^{2}$ \\
\hline Family support & 328.419 & $<.001$ & .063 & 842.755 & $<.001$ & .054 \\
\hline Teacher support & 161.335 & $<.001$ & .032 & 206.729 & $<.001$ & .014 \\
\hline Classmate support & 279.526 & $<.001$ & .054 & 263.969 & $<.001$ & .018 \\
\hline Friend support & 171.663 & $<.001$ & .034 & 612.728 & $<.001$ & .040 \\
\hline
\end{tabular}

unimportant for substance use, but only that the social support from these contexts is not a good predictor of adolescent substance use. Future research could go in depth analyzing different variables of these contexts, not only social support, to know the contextual variables involved in Spanish and Portuguese adolescents' substance use.

In contrast, social support was relevant for wellbeing, with higher values of social support implying higher adolescent well-being in both countries. Specifically, family support, teacher support and classmate support were especially relevant for higher life satisfaction in Portugal, whereas in Spain, family support and friend support were more important for life satisfaction. All the social support variables (family, teacher, classmate and friend support) were relevant for health-related quality of life in Portugal and in Spain.

Therefore, family support is a key factor for well-being in Portuguese and Spanish adolescents. In fact, parental support is the dimension most related to mental health, mainly life satisfaction (Stewart \& Suldo, 2011), as well as to lower emotional problems during adolescence (Helsen et al., 2000). It has been also found that family support is positively associated with adolescents' subjective well-being in general (Leme et al., 2015; Williams \& Anthony, 2015), and specifically with adolescent life satisfaction (Gutiérrez \& Gonçalves, 2013; Rodríguez-Fernández et al., 2012).

In addition, teacher support is relevant for life satisfaction in Portugal and for health-related quality of life in both countries, whereas friend support is relevant for life satisfaction in Spain and for healthrelated quality of life in both countries. Previous studies have found contradictory results regarding support from teachers and friends, with some studies finding that friend support (Leme et al., 2015; RodríguezFernández et al., 2012; Williams \& Anthony, 2015) and teacher support (García-Moya et al., 2015; PilkauskaiteValickiene \& Gabrialaviciute, 2015; Suldo et al., 2009) are positively associated with well-being, whereas others have not shown this association neither with friend support (Gutiérrez \& Gonçalves, 2013) nor with teacher support (Leme et al., 2015; Tummala-Narra \& Sathasivam-Rueckert, 2013). These results could explain that teacher and friend support are more relevant in Spain or Portugal depending on the well-being variable. When the relationship between social support and health-related quality of life is analyzed in this study, both teacher and friend support are relevant in both countries. This may be because health-related quality life is a more global measure of well-being than life satisfaction.

The same can happen with classmate support, because this variable, as teacher support, is more important for life satisfaction in Portugal and for health-related quality of life in both countries. Therefore support from classmates, as previous studies have found, is relevant for positive well-being (Demaray \& Malecki, 2002; Stewart \& Suldo, 2011), especially in Portugal.

These results also suggest that school-related social support is more relevant for adolescent well-being in Portugal than in Spain. Additionally, the support of classmates (not necessarily friends) in Portugal behaves similarly to the support of friends in Spain. A possible explanation is that in Spain the classmate group habitually changes every two years, whereas in Portugal the classmate group often stays together through almost all the compulsory education making it more likely that classmates are also friends and more important for adolescents in Portugal than in Spain. Nevertheless, it would be interesting to do an in-depth analysis in future research of the role of classmates and friends in Spanish and Portuguese adolescents. Furthermore, these results contribute to reflections on concerns about the education system in Spain and Portugal, as well as the data of the last HBSC international report regarding Portuguese and Spanish adolescents' perception about school compared with adolescents from other countries. In this report for 
example, Spanish adolescents were among those who reported feeling more pressured by schoolwork and Portuguese adolescents were among those who indicated liking school less (Inchley et al., 2016).

In general, as was expected taking into account cultural and political similarities, the results of this study are very similar in Portugal and Spain, probably because Spanish and Portuguese adolescents perceive their family context, peer groups and school context similarly (Serrano et al., 2004).

On the other hand, it is indispensable to indicate some limitations of this study when considering the results, such as that the HBSC study is a cross-sectional study that does not allow for establishing causal relationships. Others limitations include the fact that only one aspect, and not more dimensions, about the relationships in each context is studied and that it is examined as it is perceived by adolescents and not by other members of each context.

In conclusion, this study shows that social support is more relevant for adolescent well-being than for adolescent substance use, as well as that social support in each context, mainly family context, is important for adolescent health and well-being, which must be taken into account in interventions to promote adolescent health in Portugal and in Spain. Specifically, interventions in schools must be directed towards improving the education system considering the concerns based on adolescents' perception of school (Inchley et al., 2016). Additionally, these interventions must consider that school health promotion is one of the priorities for the school context, however the community-based model, existing in Portuguese and Spanish schools, does not always have as a main priority the school health promotion and the integration and acceptance of the health system in schools could be difficult with this model (Pommier et al., 2010), and therefore it would be necessary to overcome these limitations. Regarding interventions with families, since the economic benefits for family-related policies in Spain and Portugal are scarce (Instituto de Política Familiar, 2014) and family context was found to be the most important for adolescents in this study, it is necessary to provide more resources for Spanish and Portuguese families and, in general, to promote public policies with a family perspective across Europe (Instituto de Política Familiar, 2014), which ensure quality contexts for all families and adolescents.

\section{References}

Aleman-Diaz A., Currie D., Nic Gabhain S., Godeau E., Smith R., \& Schnohr C. W. (2013). HBSC 2013-2014 Protocol. Section 3.2. Survey instrument. Unpublished manuscript.

Bokhorst C. L., Sumter S. R., \& Westenberg P. M. (2010).

Social support from parents, friends, classmates, and teachers in children and adolescents aged 9 to 18 years: Who is perceived as most supportive? Social Development, 19, 417-426. https: / /doi.org/10.1111/j.1467-9507.2009. 00540.x

Branstetter S. A., Low S., \& Furman W. (2011). The influence of parents and friends on adolescent substance use: A multidimensional approach. Journal of Substance Use, 16, 150-160. https://doi.org/10.3109/14659891.2010.519421

Cantril H. (1965). The pattern of human concerns. New Brunswick, NJ: Rutgers University Press.

Chu P. S., Saucier D. A., \& Hafner E. (2010). Meta-analysis of the relationships between social support and well-being in children and adolescents. Journal of Social and Clinical Psychology, 29, 624-645. https://doi.org/10.1521/ jscp.2010.29.6.624

Cohen J. (1988). Statistical power analysis for the behavioral science. Hillsdale, NJ: Lawrence Erlbaum Associates.

De Goede I. H. A., Branje S. J. T., Delsing M. J. M. H., \& Meeus W. H. J. (2009). Linkages over time between adolescents' relationships with parents and friends. Journal of Youth and Adolescence, 38, 1304-1315. https:/ / doi.org/ 10.1007/s10964-009-9403-2

Demaray M. K., \& Malecki C. K. (2002). The relationship between perceived social support and maladjustment for students at risk. Psychology in the Schools, 39, 305-316. https://doi.org/10.1002/pits.10018

Erhart M., Ottova V., Gaspar T., Jericek H., Schnohr C., Alikasifoglu M., ... Ravens-Sieberer U. (2009). Measuring mental health and well-being of school-children in 15 European Countries using the KIDSCREEN-10 Index. International Journal of Public Health, 54, 160-166. https:/ / doi.org/10.1007/s00038-009-5407-7

García-Moya I., Brooks F., Morgan A., \& Moreno C. (2015). Subjective well-being in adolescence and teacher connectedness: A health asset analysis. Health Education Journal, 74, 641-654. https://doi.org/10.1177/ 0017896914555039

Gutiérrez M., \& Gonçalves T. -O. (2013). Activos para el desarrollo, ajuste escolar y bienestar subjetivo de los adolescentes [Developmental assets, school adjustment, and adolescents' subjective well-being]. International Journal of Psychology and Psychological Therapy, 13, 339-355.

Helsen M., Vollebergh W., \& Meeus W. (2000). Social support from parents and friends and emotional problems in adolescence. Journal of Youth and Adolescence, 29, 319-335. https:/ / doi.org/10.1023/A:1005147708827

Hombrados-Mendieta M. I., Gomez-Jacinto L., Dominguez-Fuentes J. M., Garcia-Leiva P., \& CastroTravé M. (2012). Types of social support provided by parents, teachers, and classmates during adolescence. Journal of Community Psychology, 40, 645-664. https:/ / doi. org/10.1002/jcop.20523

Hublet A., \& Fotiou A. (2013). HBSC 2013-2014 Protocol. Section 2 part 2. Scientific rationales: 2.10 Tobacco use. Unpublished manuscript.

Inchley J., Currie D., Young T., Samdal O., Torsheim T., Augustson L., ... Barkenow V. (2016). Growing up unequal: Gender and socioeconomic differences in young people's health and well-being. Health Behaviour in School-aged Children (HBSC) study: International report from the 2013/2014 survey. 
Copenhagen, Denmark: Health policy for children and adolescents (Report No. 7). WHO Regional Office for Europe.

Instituto de Política Familiar (2014). Informe evolución de la familia en Europa 2014 [Family evolution in Europe 2014 report]. Retrieved from IPFE webpage http:/ / www.ipfe. org /Espa\%C3\%B1a/Documentos/IPF?page=2

Jiménez-Iglesias A., Moreno C., Rivera F., \& García-Moya I. (2013).The role of the family in promoting responsible substance use in adolescence. Journal of Child and Family Studies, 22, 585-602. https://doi.org/10.1007/s10826-0139737-y

Johnson B., McBride D., Hopkins G., \& Pepper S. (2014). An examination of parent-child relationships and teen substance use: A brief report. Journal of Child \& Adolescent Substance Abuse, 23, 210-216. https://doi.org/10.1080/106 7828X.2013.786926

Kuntsche E., Simons-Morton B., De Looze M., Ter Bogt T., Godeau E., Hublet A., \& Dankulincová Z. (2013). HBSC 2013-2014 Protocol. Section 2 part 2. Scientific rationales: 2.11 Alcohol use. Unpublished manuscript.

LaRusso M. D., Romer D., \& Selman R. L. (2008). Teachers as builders of respectful school climates: Implications for adolescent drug use norms and depressive symptoms in high school. Journal of Youth and Adolescence, 37, 386-398. https://doi.org/10.1007/s10964-007-9212-4

Leme V. B. R., Del Prette Z. A. P., \& Coimbra S. (2015). Social skills, social support and well-being in adolescents of different family configurations. Paidéia, 25, 9-18. https: / / doi.org/10.1590/1982-43272560201503

Matos M. G., Simões C., Camacho I., Reis M., \& Equipa Aventura Social (2015). A saúde dos adolescentes portugueses em tempos de recessão. Dados nacionais do estudo HBSC de 2014 [Portuguese adolescents' health in times of recession. National data of the HBSC study 2014]. Lisboa, Portugal: Centro de Malária e Outras Doenças Tropicais /IHMT/ UNL \& FMH/ Universidade de Lisboa.

Moreno C., Ramos P., Rivera F., Jiménez-Iglesias A., García-Moya I., Sánchez-Queija I., ... Morgan A. (2016). Los adolescentes españoles: Estilos de vida, salud, ajuste psicológico y relaciones en sus contextos de desarrollo. Resultados del Estudio HBSC-2014 en España [Spanish adolescents: Lifestyles, health, psychological adjustment and relationships in their developmental contexts. Results of the HBSC-2014 study in Spain]. Madrid, Spain: Ministerio de Sanidad, Servicios Sociales e Igualdad.

Navarro D., Montserrat C., Malo S., González M., Casas F., \& Crous G. (2017). Subjective well-being: What do adolescents say? Child and Family Social Work, 22, 175-184. https://doi.org/10.1111/cfs.12215

Piko B. F., \& Balázs M. Á. (2012). Authoritative parenting style and adolescent smoking and drinking. Addictive Behaviors, 37, 353-356. https://doi.org/10.1016/j.addbeh. 2011.11.022

Pilkauskaite-Valickiene R., \& Gabrialaviciute I. (2015). The role of school context on subjective well-being and social well-being in adolescence. Procedia - Social and Behavioral Sciences, 191, 2588-2592. https://doi.org/ 10.1016/j.sbspro.2015.04.701
Pommier J., Jourdan D., Berger D., Vandoorne C., Piorecka B., \& de Carvalho G. S. (2010). School health promotion: Organization of services and roles of health professionals in seven European countries. European Journal of Public Health, 20, 182-188. https: / / doi.org/ 10.1093/eurpub/ckp117

Ramos P., Moreno C., Rivera F., Matos M. G., \& Morgan A. (2012). Analysis of social inequalities in health through an integrated measure of perceived and experienced health in Spanish and Portuguese adolescents. Journal of Health Psychology, 17(1), 57-67. https:/ /doi.org/10.1177/ 1359105311406154

Rasmussen M., Dür W., Freeman J., Currie D., Klinger D., Ramelow D., ... Samdal O. (2013). HBSC 2013-2014 Protocol. Section 2 part 3. Scientific rationales: 2.19 School setting. Unpublished manuscript.

Ravens-Sieberer U., \& The KIDSCREEN group Europe (2006). The KIDSCREEN Questionnaires. Quality of Life Questionnaires for Children and Adolescents. Handbook. Lengerich, Germany: Pabst Science.

Ravens-Sieberer U., Erhart M., Gosch A., Wille N., \& The European KIDSCREEN Group (2008). Mental health of children and adolescents in 12 European countries-Results from the European KIDSCREEN study. Clinical Psychology and Psychotherapy, 15, 154-163. https://doi.org/10.1002/ cpp. 574

Rodríguez-Fernández A., Droguett L., \& Revuelta L. (2012). School and personal adjustment in adolescence: The role of academic self-concept and perceived social support. Revista de Psicodidáctica, 17, 397-414.

Sánchez-Queija I., Oliva A., Parra Á., \& Camacho C. (2015). Longitudinal analysis of the role of family functioning in substance use. Journal of Child and Family Studies, 26, 232-240. https://doi.org/10.1007/s10826-015-0212-9

Serrano G., El-Astal S., \& Faro F. (2004). La adolescencia en España, Palestina y Portugal: Análisis comparativo [Adolescence in Spain, Palestine and Portugal: A comparative analysis]. Psicothema, 16, 468-475.

Stattin H., \& Kerr M. (2009). Challenges in intervention research on adolescent development. Journal of Adolescence, 32, 1437-1442. https://doi.org/10.1016/j.adolescence. 2009.10.010

Stewart T., \& Suldo S. (2011). Relationships between social support sources and early adolescents' mental health: The moderating effect of student achievement level. Psychology in the Schools, 48, 1016-1033. https: / / doi.org/10.1002/ pits. 20607

Suldo S. M., Friedrich A. A., White T., Farmer J., Minch D., \& Michalowski J. (2009). Teacher support and adolescents' subjective well-being: A mixed-methods investigation. School Psychology Review, 38(1), 67-85.

Tomé G., Matos M. G., Camacho I., Simões C., \& Diniz J. A. (2012). Portuguese adolescents: The importance of parents and peer groups in positive health. The Spanish Journal of Psychology, 15, 1315-1324. https:/ / doi.org/ 10.5209/rev_SJOP.2012.v15.n3.39417

Tummala-Narra P., \& Sathasivam-Rueckert N. (2013). Perceived support from adults, interactions with police, and adolescents' depressive symptomology: An examination of sex, race, and social class. Journal of 
Adolescence, 36(1), 209-219. https://doi.org/10.1016/ j.adolescence.2012.11.004

Williams L. R., \& Anthony E. K. (2015). A Model of positive family and peer relationships on adolescent functioning. Journal of Child and Family Studies, 24, 658-667. https: / /doi. org/10.1007/s10826-013-9876-1

Wills T. A., Resko J. A., Ainette M. G., \& Mendoza D. (2004). Role of parent support and peer support in adolescent substance use: A test of mediated effects. Psychology of Addictive Behaviors, 18, 122-134. https://doi. org/10.1037/0893-164X.18.2.122

Zimet G. D., Dahlem N. W., Zimet S. G., \&

Farley G. K. (1988). The multidimensional scale of perceived social support. Journal of Personality

Assessment, 52(1), 30-41. https://doi.org/10.1207/ s15327752jpa5201_2 